\title{
Plasmodium falciparum exposure in utero, maternal age and parity influence the innate activation of foetal antigen presenting cells Nadine Fievet ${ }^{\dagger 1,2}$, Stefania Varani ${ }^{\dagger 3,4}$, Samad Ibitokou ${ }^{1}$, Valérie Briand ${ }^{2}$, Stéphanie Louis ${ }^{6}$, René Xavier Perrin ${ }^{5}$, Achille Massougbogji ${ }^{5}$, Anne Hosmalin 6,7, Marita Troye-Blomberg ${ }^{3}$ and Philippe Deloron*2
}

\begin{abstract}
Address: ${ }^{1}$ UR010, Mother and Child Health in the Tropics, Institut de Recherche pour le Développement (IRD), Cotonou, Benin, ${ }^{2}$ UR010, IRD, IFR 71 Université René Descartes, 75006 Paris, France, ${ }^{3}$ Department of Immunology, Wenner-Gren Institute, Stockholm University, Stockholm, Sweden, ${ }^{4}$ Department of Hematology and Oncology, "L. and A. Seragnoli" University of Bologna, Bologna, Italy, ${ }^{5}$ Faculté des Science de la Santé, Cotonou, Benin, ${ }^{6}$ Institut Cochin, Université Paris Descartes, CNRS (UMR 8104), Paris, France and 7Inserm, U567, Paris, France

Email: Nadine Fievet - fievet@ird.fr; Stefania Varani - Stefania.Varani@ki.se; Samad Ibitokou - ibitokou_samad@yahoo.fr;

Valérie Briand - valerie.briand@gmail.com; Stéphanie Louis - slouis@cochin.inserm.fr; René Xavier Perrin - perrinrx@yahoo.fr; Achille Massougbogji - massougbodjiachille@yahoo.fr; Anne Hosmalin - anne.hosmalin@inserm.fr; Marita Troye-Blomberg - marita.troyeblomberg@wgi.su.se; Philippe Deloron* - Philippe.Deloron@ird.fr

* Corresponding author †Equal contributors
\end{abstract}

Published: 5 November 2009

Malaria Journal 2009, 8:25I doi:10.1186/1475-2875-8-25।
Received: 6 May 2009

Accepted: 5 November 2009

This article is available from: http://www.malariajournal.com/content/8/I/25।

(C) 2009 Fievet et al; licensee BioMed Central Ltd.

This is an Open Access article distributed under the terms of the Creative Commons Attribution License (http://creativecommons.org/licenses/by/2.0), which permits unrestricted use, distribution, and reproduction in any medium, provided the original work is properly cited.

\begin{abstract}
Background: Malaria in pregnancy is associated with immunological abnormalities in the newborns, such as hampered T-helper I responses and increased T-regulatory responses, while the effect of maternal Plasmodium falciparum infection on foetal innate immunity is still controversial.
\end{abstract}

Materials and methods: The immunophenotype and cytokine release by dendritic cells (DC) and monocytes were evaluated in cord blood from 59 Beninese women with or without malaria infection by using flow cytometry.

Results: Accumulation of malaria pigment in placenta was associated with a partial maturation of cord blood myeloid and plasmacytoid DC, as reflected by an up-regulated expression of the major histocompatibility complex class II molecules, but not CD86 molecules. Cells of newborns of mothers with malaria pigment in their placenta also exhibited significantly increased cytokine responses upon TLR9 stimulation. In addition, maternal age and parity influenced the absolute numbers and activation status of cord blood antigen-presenting cells. Lastly, maternal age, but not parity, influenced TLR3, 4 and 9 responses in cord blood cells.

Discussion: Our findings support the view that placental parasitization, as indicated by the presence of malaria pigment in placental leukocytes, is significantly associated with partial maturation of different DC subsets and also to slightly increased responses to TLR9 ligand in cord blood. Additionally, other factors, such as maternal age and parity should be taken into consideration when analysing foetal/neonatal innate immune responses.

Conclusion: These data advocate a possible mechanism by which PAM may modulate foetal/ neonatal innate immunity. 


\section{Background}

Pregnancy-associated Plasmodium falciparum malaria (PAM) results, sometimes, in massive intervillous inflammation that contributes to placental insufficiency, impaired intra-uterine growth and consequently to low birth weight in the newborns and a higher risk of dying early in life [1-4].

Infants born to women with PAM are more predisposed to $P$. falciparum infection in their first year of life [5-7]. Immunological mechanisms are generally considered to play an important role in causing this susceptibility. In utero sensitization to transplacentally transferred soluble $P$. falciparum antigens may constitute the basis for increased susceptibility to malaria episodes in early life. Importantly, it has been demonstrated that cord blood mononuclear cells (CBMC) of neonates born to mother with PAM specifically respond to plasmodial asexual stage antigens, and that cord blood B cells produce anti-malaria specific IgM and IgE antibodies [5,8-10], providing irrefutable evidence of in utero sensitization.

In this context, active infection in the placenta by $P$. falciparum was associated with hampered T-helper 1 (Th1) responses, as reflected by reduced IFN- $\gamma$ production upon T-cell stimulation [9]. In addition, the anti-inflammatory IL-10 cytokine is more frequently produced by CBMC of those born to mothers with PAM compared with noninfected mothers [11]. CD4 ${ }^{+} \mathrm{CD} 25^{\text {high }}$ regulatory T-cells (Treg) are a principal source of IL-10 in such cases [12]. Treg are found at higher frequency in cord blood (CB) of neonates born to mothers with PAM at delivery as compared to unexposed newborns [12].

Because of their key function in the initiation and regulation of adaptive immune responses, it is reasonable to assume that antigen presenting cells (APC), such as monocytes and dendritic cells (DC), contribute to the modulation of foetal immune responses upon exposure to $P$. falciparum in utero. Indeed, DC seem to play an important role in both protective and dysfunctional immune responses against malaria in murine models $[13,14]$. DC comprise a heterogeneous population of cells; myeloid DC (MDC) that orchestrate T-cell responses through a fine modulation of IL-12 secretion, while plasmacytoid DC (PDC) are an essential component of innate and adaptive immunity through secretion of type I interferons (IFN) in response to pathogens [15]. A minor blood MDC population, blood DC antigen (BDCA)-3+ cells, has been described sharing the same ontogeny as the more frequent BDCA-1+ MDC subset [16,17].

The foetal/neonatal immune system exhibits quantitative and functional differences from the adult one and neonatal DC have reduced ability in delivering co-stimulatory signals to T-cells as a consequence of their incomplete maturation [18]. They also exhibit a markedly decreased capacity in secreting IL-12 and IFN- $\alpha[19,20]$. This probably contributes to the development and relative predominance of Treg in CB [21], although seemingly less marked in Africans vs. Europeans [22].

Whether and how $P$. falciparum infection in the mother may affect foetal innate immunity is poorly understood. One study conducted in The Gambia reported lower lipopolysaccharide (LPS)-induced IFN- $\gamma$ and IL-12 activity in CBMC of newborns of mothers with PAM as compared to uninfected mothers [9]. A more recent study revealed that CBMC of neonates born of Gabonese mothers with $P$. falciparum infection exhibit significantly increased IFN- $\gamma$ responses upon stimulation with toll-like receptor (TLR)3 and TLR4 ligands [22].

Contrasting findings have also been reported on the characterization of DC subsets in CBMC of neonates born to $P$. falciparum-infected mothers. One study reported a significantly higher frequency of MDC [23], while another reported profoundly reduced numbers of PDC [24] as compared to unexposed newborns.

The mechanistic hypothesis behind the present study is that malaria infection in the mother may cause a dysfunctional activation of foetal APC by parasite-derived products that cross the placenta. An altered activation of foetal APC could be responsible for the impaired T-cell response that is observed in infants born to mothers with PAM.

Using flow cytometry, subpopulations of DC and monocytes were evaluated in $\mathrm{CB}$ of neonates from Beninese women with or without malaria infection. In addition, the impact of $P$. falciparum exposure in utero, on the innate activation of foetal APC was examined by stimulating CBMC with specific TLR ligands; LPS was employed to activate TLR4 on monocytes and BDCA- $1+\mathrm{MDC}$, polyinosine-polycytidilic acid (PolyI:C) to selectively stimulate TLR3 expressed in MDC, and CPG-A ODN to specifically activate TLR9 expressed in PDC $[16,17,25]$.

\section{Methods \\ Study population}

Pregnant women were enrolled after informed consent from July 2006 to January 2007; in the Hospital "Mother and Child Lagune", the main obstetrical referring hospital in Cotonou. This study was approved by the Science and Health Faculty Ethics Committee. To identify women with malaria infection, a rapid immuno-chromatographic test (Cypress $^{\circledast}$, Langdorp, Belgium) was performed on fingerpricked capillary blood before delivery. Thirty $P$. falciparum-infected women and twenty-nine uninfected women matched for parity and age were enrolled in the study. Twenty-five ml heparinized CB were collected immediately after delivery. According to national policy, 
pregnant women receive intermittent preventive treatment with sulphadoxine-pyrimethamine (SP). Despite this usage of SP was declared by only $47 \%$ of the women, while the remaining mothers declared having taken chloroquine (CQ) as chemoprophylaxis.

\section{Determination of $\mathbf{P}$. falciparum status of the mothers at delivery}

Thin and thick smears were prepared from maternal peripheral, placental intervillous and cord blood, stained with Giemsa and examined for the presence and density of parasites. Malaria infection in the mothers at delivery was defined by the presence of parasites in the placental and/or maternal peripheral blood. The presence of malaria pigment (MP) was also evaluated in leukocytes of placental intervillous blood (Table 1).

\section{CBMC cultures}

Mononuclear cells were isolated from CB by centrifugation over Ficoll-Hypaque (Pharmacia Uppsala, Sweden). Cells were washed twice and resuspended in RPMI 1640 medium with L-glutamine (Gibco Eragny, France) supplemented with $10 \%$ foetal bovine serum (FBS, Gibco) and $50 \mu \mathrm{g} / \mathrm{ml}$ gentamycin to a final concentration of $2 \times 10^{6}$ CBMC/ml. Viability was $>99 \%$ in all tested samples as determined by Trypan blue staining.

To assess production of IL-12, CBMC were stimulated for 8 hours with LPS $(100 \mathrm{ng} / \mathrm{ml}$; Sigma Aldrich, St. Louis MO), PolyI:C $(20 \mu \mathrm{g} / \mathrm{ml}$; Sigma Aldrich), or synthetic haemozoin $(\mathrm{Hz}, 5 \mu \mathrm{g} / \mathrm{ml})$ in the presence of Brefeldin-A (BD Pharmingen, San Diego, CA) during the last five hours of incubation. Hz was prepared from haemin chloride as described [26]. Endotoxin levels in the Hz preparation were found to be below the threshold $(<0.125$ units/ $\mathrm{ml}$ ) by the Limulus-amoebocyte lysate assay (Biowhittaker, Cambrex). To assess cytokine production by CBMC upon contact with TLR9 ligands, CpG-A ODN $2216(3 \mu \mathrm{g} /$ $\mathrm{ml}$; Metabion $\mathrm{GmbH}$, Martinsried, Germany) was employed.

\section{Immunophenotype of APC}

CBMC were resuspended in staining buffer (PBS 2\% FBS, $5 \mathrm{mM}$ EDTA). Cells were first incubated with FcR blocking reagent (Miltenyi Biotech, Bergisch-Gladbach, Germany) and then with anti-CD14-FITC, anti-CD19-FITC, antiBDCA-1-PE, anti-BDCA-2-PE, anti-BDCA-3-PE (Miltenyi Biotech), anti-HLA-DR-PerCP and anti-CD86-APC (BD Pharmingen), or alternatively mouse isotype controls (BD Pharmingen). Cell acquisition was performed with a FACSCalibur flow cytometer (BD Pharmingen) and analysis was performed by CellQuest software as described in Figure $1 \mathrm{~A}$.

\section{Intracellular cytokine staining for IL- I 2}

After stimulation, cells were incubated with FCR blocking reagent and stained with anti-HLA-DR-PerCP, anti-CD14FITC, anti-CD19-FITC, anti-BDCA-1-PE, anti-BDCA-3-PE or isotype controls for $10 \mathrm{~min}$ at $4{ }^{\circ} \mathrm{C}$. Cells were then fixed with FACS lysing solution, washed and incubated in a permeabilization buffer (staining buffer with $0.25 \%$ saponin and $5 \% \mathrm{AB}$ human serum) for $15 \mathrm{~min}$ at $4^{\circ} \mathrm{C}$. After centrifugation, cells were stained with anti-IL-12APC (BD Pharmingen) or alternatively APC-conjugated

Table I: Summary of the study population.

\begin{tabular}{|c|c|c|}
\hline \multirow[b]{2}{*}{ Characteristics } & \multicolumn{2}{|c|}{ Study group } \\
\hline & P. falciparum-positive & P. falciparum-negative \\
\hline Number of subjects $n=59$ & 30 & 29 \\
\hline Age of mother, mean $\pm S D$, years & $25.4 \pm 6.1$ & $26.3 \pm 5.2$ \\
\hline Pregnancies, no., mean $\pm S D$ & $2.2 \pm 1.5$ & $2.1 \pm 1.1$ \\
\hline I-2 pregnancies $(n=4 I)$ & 21 & 20 \\
\hline$\geq 3$ pregnancies $(n=18)$ & 9 & 9 \\
\hline Ratio of malaria prevention (CQ/SPa) (30/27) & $19 / 10$ & $11 / 17$ \\
\hline Declaration of malaria infection during pregnancy (\%) & 33.3 & 3.7 \\
\hline Reported use of bednet (\%) & 83.3 & 82.8 \\
\hline Neonate birth weight, mean $\pm \mathrm{SD}, \mathrm{g}$ & $3052.8 \pm 443.3$ & $3059.6 \pm 412.5$ \\
\hline Neonate gender, female/male (2I/34) & $10 / 19$ & $11 / 15$ \\
\hline \multicolumn{3}{|l|}{ P. falciparum density at delivery: } \\
\hline peripheral blood, mean $\pm \mathrm{SD}, \mathrm{iRBC} b / \mu \mathrm{l}$ & $19,037 \pm 55,257$ & 0 \\
\hline intervillous blood, mean $\pm \mathrm{SD}, \mathrm{iRBC} / \mu \mathrm{l}$ & $245,764 \pm 475,906$ & 0 \\
\hline cord blood, mean $\pm S D, i R B C / \mu l$ & 0 & 0 \\
\hline intervillous blood leukocytes with MPc, $n=59$ & 19 & 0 \\
\hline
\end{tabular}

a CQ/SP; Chloroquine/sulfadoxine-pyrimethamine

b iRBC; infected red blood cells

c MP; malaria pigment 


\section{A}

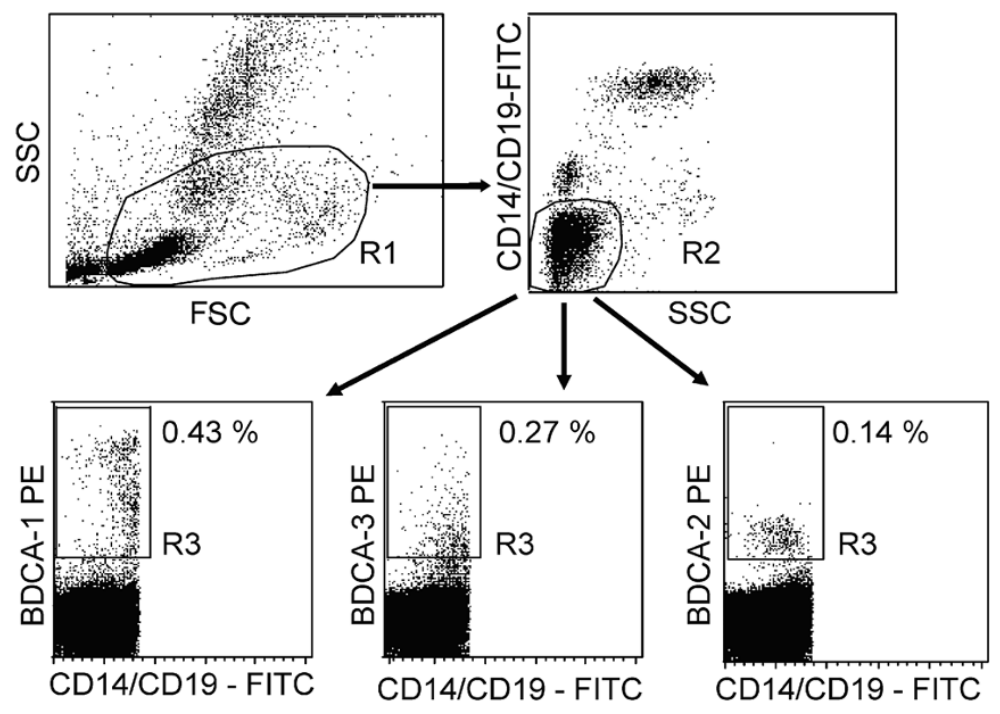

B

$\square \mathrm{MP}^{-}$placenta

$\mathbb{M P}{ }^{+}$placenta
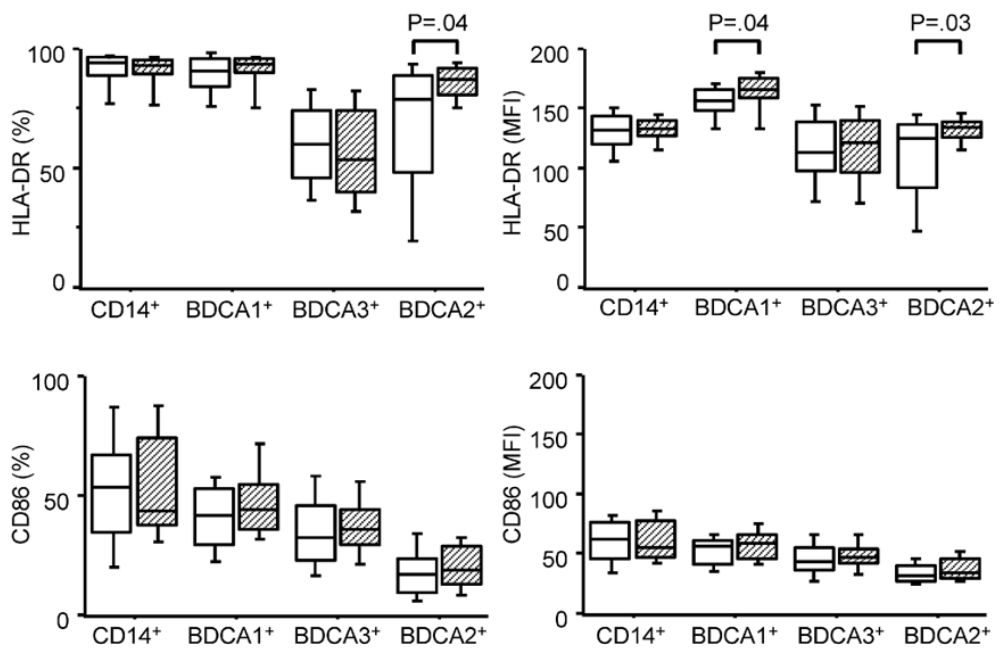

\section{Figure I}

(A) Flow cytometric identification of MDC (BDCA- I $^{+}$or BDCA-3+) and PDC (BDCA-2+) in CBMC from one subject. RI gate was set to include only viable mononuclear cells, as determined by forward scatter (FSC) and side-scatter (SSC) characteristics. Furthermore, cells were gated to be both CDI4 and CDI9 negative (R2) and either BDCA-I, BDCA-3, or BDCA-2 positive (R3). One million events were analysed for DC, 300,000 events for monocytes and 100,000 events for isotype control enumeration. The percentages represent relative levels of the different DC populations from the selected subject. (B) Partial activation of cord blood BDCA-I+ and BDCA-2+ cells is related to the presence of MP in placenta. The expression levels of HLA-DR and CD86 were measured by flow cytometry on foetal APC subsets from 16 MP-positive (diagonal striped bars) and 39 MP-negative mothers (white bars). Boxplots illustrate the medians and the $25^{\text {th }}$ and $75^{\text {th }}$ percentiles. $Y$-axes report percentage of positive cells and mean fluorescence intensity (MFI) for the activation markers CD86 and HLA-DR in different APC subsets. P-values were calculated by Mann-Whitney $U$ test. The significance limit was $P<0.05$. 
isotype control for $30 \mathrm{~min}$ at $4{ }^{\circ} \mathrm{C}$, and then analysed by flow cytometry. BDCA- ${ }^{+}$and BDCA- $3^{+}$cells that did not express CD19 and CD14 were gated together as MDC.

\section{Determination of cytokine levels in plasma samples and supernatants}

IFN- $\alpha$ levels were measured with an ELISA kit (PBL Biomedical Laboratories, Piscataway, NJ). The assay sensitivity was $12.5 \mathrm{pg} / \mathrm{ml}$. A panel of pro-inflammatory and antiinflammatory cytokines including IL-6, IL-10, IL-12, (MIP)-1 $\alpha /$ CCL3; TNF- $\alpha$ and IFN- $\gamma$ were quantified by the Human Cytokine Cytometric Bead Array Kit (BD Pharmingen) using flow cytometry. The assay sensitivity was $1.6 \mathrm{pg} / \mathrm{ml} ; 0.13 \mathrm{pg} / \mathrm{ml} ; 0.6 \mathrm{pg} / \mathrm{ml} ; 0.2 \mathrm{pg} / \mathrm{ml} ; 1.2 \mathrm{pg} /$ $\mathrm{ml}$; and $1.8 \mathrm{pg} / \mathrm{ml}$ for IL-6, IL-10, IL-12, MIP-1- $\alpha / C C L 3$, TNF- $\alpha$ and IFN- $\gamma$ respectively. Results were formatted using the BD CBA Analysis Software.

\section{Statistical analysis}

Background values on cytokines in supernatants obtained from unstimulated cells were subtracted from data acquired from cultures in the presence of stimuli. Normally distributed variables were analysed by unpaired $t$ test. Data that were not normally distributed even after log-transformation were analysed by the non-parametric Mann-Whitney test. To test if the age of the mother was related to parity, Spearman rank correlation was employed. Linear regression analysis on log-transformed data was used to identify dependent variables for a multivariate analysis. The significance limit was $\mathrm{P}<0.05$.

\section{Results}

Partial activation of foetal DC is related to the presence of MP in placenta

The absolute numbers of APC subpopulations and expression of activation markers are described in Table 2. CBMC contained $\mathrm{MDC}$ at a higher frequency than PDC, resulting in a mean BDCA-1+/BDCA-2+ cell ratio of $4.4 \pm 5.2$.
Segregation on the basis of maternal malaria infection (i.e. presence of parasites in placental and/or maternal peripheral blood) did not show differences either in the absolute number of foetal APC or in the expression levels of MHC class II and CD86 molecules in different APC subsets studied (additional file 1). However, foetal APC status segregated on the basis of presence or absence of $\mathrm{MP}$ in the placenta, revealed a significant up-regulation of the MHC-class II expression, but not of CD86, on BDCA$1^{+}$and BDCA-2+ DC in CB obtained from MP-positive mothers as compared to MP-negative mothers (Figure $1 \mathrm{~B})$. Thus, a partial activation of foetal DC is related to the presence of MP in the placenta, and not to maternal infection at delivery.

\section{Impact of age and parity of the mother on the frequency and activation status of foetal APC}

The absolute numbers of monocytes and BDCA-1+ MDC in $\mathrm{CB}$ were negatively associated with maternal age and parity (Figure $2 \mathrm{~A}$ and $2 \mathrm{~B}$ ). In addition, maternal age showed a positive correlation with higher expression levels of CD86 on monocytes (Figure 2A). We also observed significantly increased MHC-class II expression on BDCA$2^{+} \mathrm{DC}$ in $\mathrm{CB}$ from multigravidae as compared to mothers undergoing first or second pregnancy (Figure 2B).

As expected, maternal age and multiparity were related (Spearman coefficient $=0.47 ; \mathrm{P}=0.0002$ ) . The multivariate analysis showed an effect of both age and parity on $\mathrm{CB}$ monocytes and BDCA- $1^{+}$cells absolute numbers (age: $\beta=$ $+0,55(0,10-1,00)$ with $P=0,02$ and parity: $\beta=+0,42(-$ $0,06-0,90)$ with $P=0,08$ ).

\section{Malaria status of the mother does not affect immunophenotype of foetal APC upon TLR and $\mathrm{Hz}$ stimulation}

By examining MHC class II expression, CB monocytes and MDC were activated by LPS and PolyI:C stimulation

Table 2: APC absolute numbers and immunophenotype in cord blood samples.

\begin{tabular}{|c|c|c|c|c|}
\hline \multirow[b]{2}{*}{ Parameter: median ( \pm interquartile) } & \multicolumn{4}{|l|}{ APC subset } \\
\hline & Monocytes & MDC & & PDC \\
\hline & CDI4+ & BDCA- I $^{+}$ & BDCA-3 $^{+}$ & BDCA-2 $^{+}$ \\
\hline$\%$ of total CBMC & $13.00 \pm(7.10)$ & $0.62 \pm(0.42)$ & $0.19 \pm(0.18)$ & $0.25 \pm(0.18)$ \\
\hline absolute no./ml & $624,787 \pm(571,630)$ & $23,850 \pm(30,430)$ & $8,160 \pm(12,225)$ & $9,680 \pm(9,032)$ \\
\hline$\%$ HLA-DR ${ }^{+}$cells & $93.87 \pm(7.09)$ & $93.53 \pm(11.01)$ & $59.95 \pm(28.55)$ & $82.64 \pm(31.69)$ \\
\hline HLA-DR, MFIa & $|32.7| \pm(17.69)$ & $162.50 \pm(18.81)$ & $1 \mid 8.26 \pm(40.8 I)$ & $129.9 \pm(39.59)$ \\
\hline$\% \mathrm{CD}^{6} 6^{+}$cells & $50.28 \pm(34.37)$ & $44.67 \pm(22.15)$ & $35.95 \pm(20.98)$ & $18.47 \pm(|3.4|)$ \\
\hline CD86, MFI & $62.36 \pm(30.17)$ & $57.51 \pm(21.12)$ & $45.54 \pm(17.80)$ & $33.29 \pm(13.77)$ \\
\hline
\end{tabular}

Immunophenotyping was performed on 55 cord blood samples; 27 from malaria infected women and 28 from uninfected women. APC were quantified as a percentage of the total CBMC. Absolute numbers of MDC, PDC and monocytes were calculated from CBMC counts. HLA-DR and CD86 expression levels were then examined in different APC subsets.

a; MFI, mean fluorescence intensity 

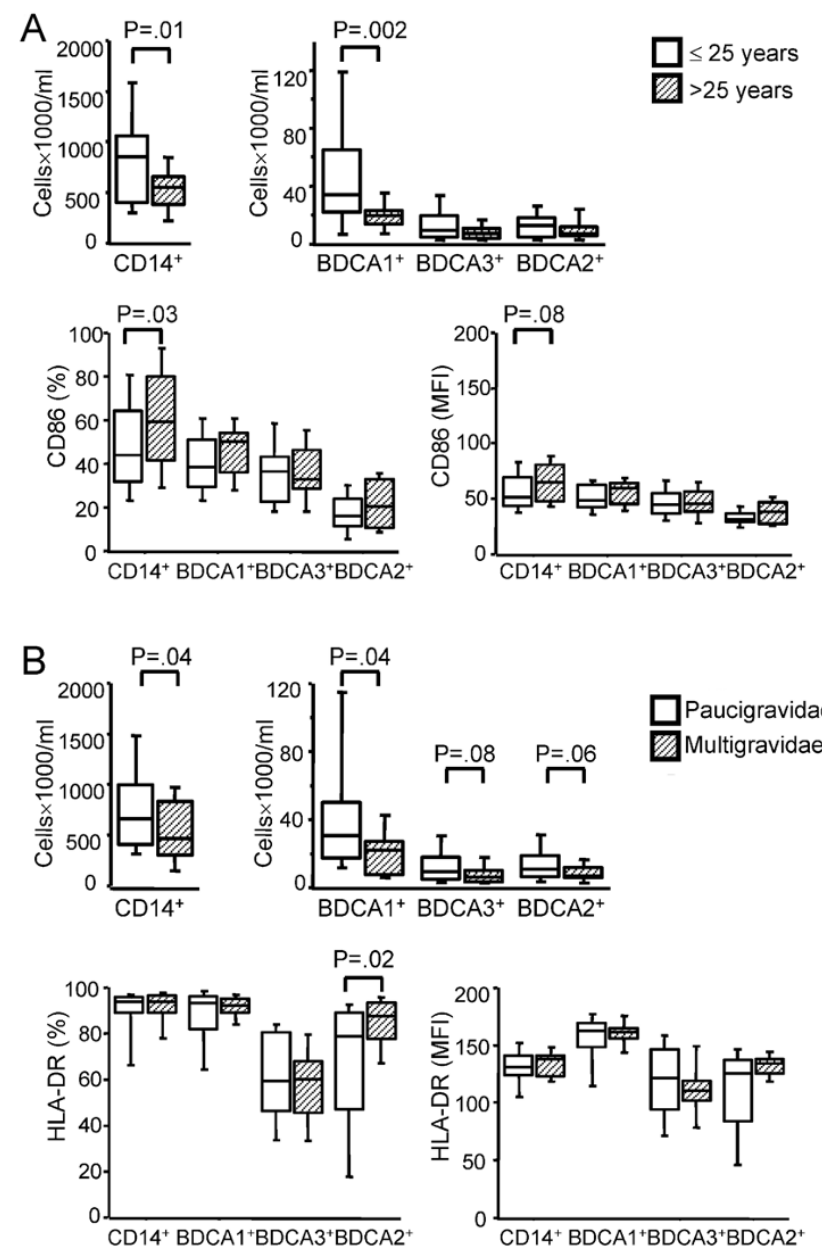

Figure 2

Association between APC numbers and activation and maternal age or parity. (A) Data were segregated into 2 groups according to the median value for maternal age. Absolute numbers and CD86 expression levels (as percentage of positive cells and mean fluorescence intensity, MFI) on different foetal APC subsets were analysed as a function of maternal age in 31 women $\leq 25$ years of age and 24 women > 25 years of age Boxplots illustrate the medians and the $25^{\text {th }}$ and $75^{\text {th }}$ percentiles. (B) Women were divided for parity as paucigravidae (Ist and 2nd pregnancy) and multigravidae ( $\geq 3$ pregnancies) as we previously observed that women at first and second pregnancy exhibited the same risk of malaria infection (Fievet, unpublished data). Absolute numbers and HLA-DR expression levels (as percentage of positive cells and mean fluorescence intensity, MFI) on different foetal APC subsets were analysed as a function of parity in 37 women undergoing first or second pregnancy and 18 multigravidae. Boxplots illustrate the medians and percentiles. Pvalues were calculated by Mann-Whitney $U$ test. The significance limit was $P<0.05$.

(Table 3). In addition, when we added synthetic $\mathrm{Hz}$ as stimulus, we found monocytic activation, as shown by significantly increased expression levels of MHC class II molecules, while the MHC class II levels on MDC were not affected (Table 3). However, there was no difference in MHC class II expression following stimulation with TLR3 and TLR4 ligands or synthetic Hz on CB monocytes and MDC when segregated according to the presence of MP in the placentas (Table 3). Thus, MP positivity did not affect further phenotypic ex- vivo maturation of foetal APC by diverse TLR ligands or $\mathrm{Hz}$.

\section{Plasmodium falciparum infection in the mother induces amplification of TLR9 response in foetal leukocytes}

Cytokine secretion in APC is triggered by the recognition of microbial pathogens through TLR [15]. The ability of foetal APC to secrete cytokines by stimulating CBMC with different TLR ligands was investigated. IFN- $\alpha$ was evaluated in supernatants of unstimulated, CpG-A- and Hzstimulated CBMC cultures. As previously reported $[19,27]$, unstimulated and synthetic Hz-stimulated CBMC did not secrete any IFN- $\alpha$, while CpG-A-stimulated cells, most likely PDC, produced low levels of this cytokine $(0.12 \pm 13.79 \mathrm{pg} / \mathrm{ml}$; median \pm interquartile $)$. No difference was observed in IFN- $\alpha$ production upon CpG-A stimulation when CBMC were segregated according to accumulation of MP in placenta: $(0.01 \pm 12.04 \mathrm{pg} / \mathrm{ml}$ vs $1.90 \pm 18.21 \mathrm{pg} / \mathrm{ml}$; MP-negative vs MP-positive women; $\mathrm{p}=0.45)$.

The levels of IL-6, IL-10, IL-12, MIP-1 $\alpha /$ CCL3 $\alpha$ IFN- $\gamma$ and TNF- $\alpha$ were also quantified in supernatants of CBMC that were either unstimulated or stimulated with synthetic $\mathrm{Hz}$ or different TLR ligands. TLR3, TLR4 and, to a lesser extent, TLR9 stimulation induced release of both pro- and anti-inflammatory cytokines (Figure 3 ). The presence of $\mathrm{MP}$ in the placenta influenced TLR-mediated CBMC cytokine responses such that the production of IL-10 and TNF- $\alpha$ was significantly higher upon TLR9 stimulation and there was a similar trend for increased production of IFN- $\gamma$ after TLR-3 stimulation in CBMC of neonates of mothers with MP-positive placenta as compared to MPnegative mothers (Figure 3 ).

To further analyse the production of IL-12 by different APC subsets upon TLR3 and TLR4 stimulation, we performed intracellular cytokine staining. Only a minor population of foetal APC produced IL-12. In fact, $2.7 \% \pm 3.4 \%$ and $2.6 \% \pm 3.8 \%$ of IL-12-producing monocytes (median \pm interquartile) were observed in response to LPS and PolyI:C, respectively, and $2.7 \% \pm 3.5 \%$ and $2.0 \% \pm 3.6 \%$ of IL-12-producing MDC (median \pm interquartile) were found in responses to LPS and PolyI:C, respectively. However, no differences were observed when comparing foetal APC of mothers with or without MP accumulation in placenta (table 4).

Fifty-seven plasma samples from CB of mothers were analysed for cytokine levels. In all samples we found detecta- 
Table 3: Immunophenotype of fetal APC upon TLR and Hz stimulation.

\begin{tabular}{|c|c|c|c|c|c|c|c|}
\hline \multirow[b]{2}{*}{$\begin{array}{l}\text { Parameter } \\
\text { median } \\
( \pm \text { interquartile) }\end{array}$} & \multirow[b]{2}{*}{ stimulus } & \multirow[b]{2}{*}{ Total CB } & \multicolumn{2}{|l|}{ Monocytes } & \multicolumn{2}{|r|}{ MDC } & \multirow[b]{2}{*}{ MP-Positive } \\
\hline & & & MP-Negative & MP-Positive & Total CB & MP -Negative & \\
\hline \multirow[t]{4}{*}{$\% \mathrm{HLA}^{-\mathrm{DR}^{+}}$cells } & unstimulated & $89.34 \pm(13.90)$ & $89.33 \pm(16.57)$ & $89.79 \pm(7.27)$ & $88.78 \pm(19.83)$ & $87.33 .40 \pm(19.83)$ & $92.62 \pm(20.13)$ \\
\hline & $\mathrm{Hz}$ & $91.03 \pm(10.10)$ & $90.46 \pm(11.97)$ & $92.27 \pm(8.31)$ & $90.99 \pm(12.22)$ & $89.40 \pm(13.55)$ & $91.37 \pm(7.88)$ \\
\hline & LPS & $96.73 \pm(6.22)$ & $96.73 \pm(7.6 I)$ & $96.45 \pm(3.14)$ & $93.24 \pm(11.08)$ & $93.24 \pm(11.66)$ & $93.38 \pm(15.23)$ \\
\hline & Poly I:C & $96.79 \pm(4.68)$ & $96.79 \pm(7.99)$ & $96.70 \pm(2.27)$ & $93.99 \pm(12.57)$ & $93.08 \pm(9.54)$ & $92.09 \pm(16.42)$ \\
\hline \multirow[t]{4}{*}{ HLA-DR, MFla } & unstimulated & $|20.8| \pm(20.14)$ & $121.15 \pm(24.36)$ & $119.54 \pm(16.48)$ & $143.85 \pm(23.19)$ & $143.57 \pm(21.28)$ & $149.29 \pm(22.77)$ \\
\hline & $\mathrm{Hz}$ & $\begin{array}{l}122.08 \pm(20.42) \\
*\end{array}$ & $125.36 \pm(23.00)$ & $121.47 \pm(15.15)$ & $146.96 \pm(14.93)$ & $151.30 \pm(14.69)$ & $156.06 \pm(20.47)$ \\
\hline & LPS & $135.72 \pm(16.78)^{*}$ & $137.56 \pm(18.02)$ & $133.23 \pm(13.82)$ & $152.40 \pm(16.28)^{*}$ & $145.49 \pm(19.31)$ & $150.67 \pm(13.84)$ \\
\hline & Poly I:C & $136.53 \pm(15.66)^{*}$ & $137.27 \pm(19.29)$ & $136.4 \mid \pm(|3.5|)$ & $150.16 \pm(14.93)^{*}$ & $150.61 \pm(16.72)$ & $149.60 \pm(16.35)$ \\
\hline
\end{tabular}

Immunophenotyping on stimulated cells was performed on 43 cord blood samples (total CB); 12 from women with MP accumulation in placenta (MPPositive) and 31 without MP in placenta (MP-negative). All comparison between MP-Negative and MP-Positive were not significant.

a; MFI, mean fluorescence intensity

*; $\mathrm{P}<0.05$

ble levels of IFN- $\alpha$, IL-6, IL-10, IL-12, MIP-1- $\alpha /$ CCL3 and TFN- $\gamma$ but no IFN- $\gamma$. The CB plasma levels of the different cytokines were not influenced by the presence of malaria infection in the mother (Figure 4A) or by MP accumulation in placenta (Figure 4B).

Thus, cytokine responses to TLR3, 4 and 9 ligands and to $\mathrm{Hz}$ were observed, with amplification of TLR9-mediated responses in CBMC from MP-positive mothers.

\section{Maternal age influences TLR3, 4 and 9 responses of foetal leukocytes and cytokine levels in foetal plasma}

Segregation of samples into two groups according to the median value for maternal age revealed significant differences in cytokine production after TLR3, 4 and 9 stimulation of CBMC. The levels of TNF- $\alpha$, IFN- $\gamma$, MIP-1 $\alpha /$ CCL3 and IL-10 produced in response to LPS by CBMC of newborns of mothers $\leq 25$ years were significantly higher than those produced by CBMC of neonates born to mothers > 25 years. In addition, the amount of TNF- $\gamma$ and IL-10 produced by CBMC in response to TLR3 and TLR9 ligands were negatively associated with maternal age (Figure 5). Parity had no influence on cytokine secretion by CBMC upon TLR stimulation (Figure 6).

Significant differences were also observed in CB cytokine levels according to maternal age. Foetal plasma from mothers $>25$ years of age exhibited significant lower levels of IL-10 as compared to younger mothers (Figure 4C). Thus, productions of cytokines by CBMC and plasma levels for IL-10 were higher in children born to the younger mothers.
When an effect of both MP-positivity in placenta and maternal age on cytokines in supernatants was observed, the multivariate analysis showed that maternal age was predominant and that effect of MP-positivity disappeared for IL-10 in response to TLR9 ligands $(\beta=1.23(0.56$ 1.89) $\mathrm{P}=0.001)$. However, both MP-positivity and maternal age had an effect on TNF- $\alpha$ secretion upon TLR9 stimulation (MP: $\beta=0.88$ (0.024-1.74), $P=0.04$; Age: $\beta=1.04$ $(0.23-1,74), P=0.04)$.

\section{Discussion}

The consensus view is that in utero sensitization to $P$. falciparum antigens is a common phenomenon during PAM. Parasites do not usually cross the placental barrier and such sensitization is most probably caused by the transplacental passage of soluble $P$. falciparum antigens $[8,28]$. Accordingly, no parasite-positive smears were detected in CB samples of malaria-infected women in this study population.

Pregnant women declared to receive either SP or CQ as malaria prevention during pregnancy. In contrast to a previous study performed in the same area [29], no differences were observed for $P$. falciparum infection rate according to the type of prophylaxis used by the mothers. However, the number of subject included in this study was low and the project was not designed to examine this matter.

In this study, the foetal BDCA-1+ and BDCA-2+ DC subsets expressed significantly higher levels of MHC class II molecules upon PAM, as indicated by the presence of $P$. falciparum MP in placenta, which is in agreement with 
IL-6
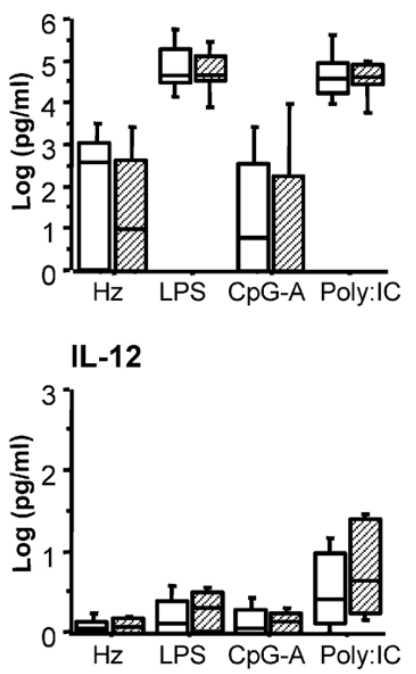

TNF- $\alpha$

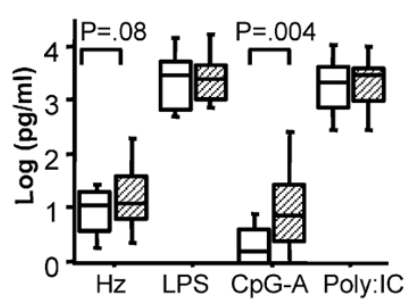

IL-10
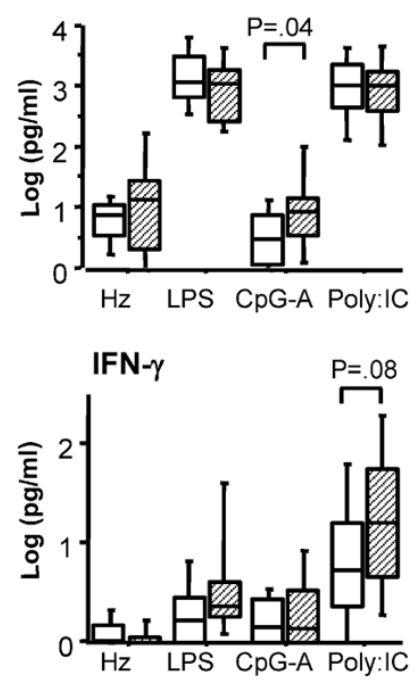

MIP-1 $\alpha$

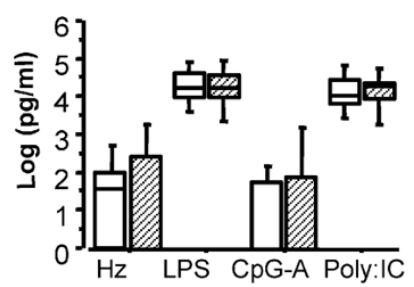

Figure 3

TLR-induced cytokine responses in CBMC obtained from women with or without MP accumulation in placenta. CBMC $(2 \mathrm{million} / \mathrm{ml})$ were stimulated or not with synthetic $\mathrm{Hz}(5 \mu \mathrm{g} / \mathrm{ml})$, LPS (I $00 \mathrm{ng} / \mathrm{ml})$, CpG-A $(3 \mu \mathrm{g} / \mathrm{ml})$ or Polyl:C $(20 \mu \mathrm{g} / \mathrm{ml})$. After 18 hours, supernatants were collected and analysed for IL-6, IL-I0, IL-I2, IFN- $\alpha$, TNF- $\alpha$ and MIP-I $\alpha / C C L 3$ levels. Data represent median values and percentiles for 59 individuals; 16 MP-positive (diagonal striped bars) and 43 MP-negative mothers (white bars). Cytokine levels in unstimulated cells were subtracted from the values shown. P-values were calculated by Mann-Whitney $U$ test or $t$ test (see Subjects, Materials and Methods). The significance limit was $P<0.05$.

previous data [23]. The observation that CD86 expression on foetal DC was unaffected by PAM suggests that $P$. falciparum stimulation in utero induces only partial activation of these cells. Failure to provide DC with a sufficiently strong costimulatory signals can impair the ability to form stable interactions with T-cells, as recently shown in a murine model of malaria [30]. Partial DC maturation can lead to altered T-cell activation and induction of tolerance [31-34], possibly contributing to impaired immune responses that have been observed in the offspring of mothers with PAM $[9,11]$.

The findings presented in this study diverge from those of studies on peripheral blood DC from children with acute malaria, where expression levels of MHC class II on the BDCA-1+ DC are reduced compared to healthy controls $[35,36]$. Also, no increase in foetal BDCA-3+ DC was detected upon maternal malaria infection in this study like others have shown in children with severe malaria [35]. Circulating APC are continuously exposed to $P$. falciparum-infected erythrocytes during malaria episodes in children, which may exert a contact-mediated inhibitory effect on DC functionality, as demonstrated by in vitro studies [4,37]. Conversely, infected erythrocytes are rarely detected in $\mathrm{CB}$ of those born to mothers with PAM $[23,38]$. Thus, foetal APC would rarely if ever encounter parasitized red-blood cells, but would be primarily exposed to and influenced by parasite-derived soluble compounds.

Interestingly, TLR9 stimulation led to increased pro- and anti-inflammatory responses of $\mathrm{CBMC}$ of neonates whose mothers had MP accumulating in placentas, and there was a tendency towards increased IFN- $\gamma$ response upon TLR3 stimulation in the same group. Responses via other TLR ligands, such as LPS were amplified in CBMC but did not change appreciably as a function of maternal malaria infection. Thus, foetal different TLR responses are independently modulated by in utero exposure to $P$. falciparum, consistent with a recent study [22].

In humans only PDC and B cells express TLR9 [39]. In this study, CPG-A, a TLR9 ligand that specifically stimulates

Table 4: Accumulation of MP in placenta does not affect IL-I 2 production by APC upon TLR3 and TLR4 stimulation.

\begin{tabular}{llllll}
\hline & & Monocytes & & MDC \\
\cline { 3 - 6 } $\begin{array}{l}\text { Parameter } \\
\text { median }( \pm \text { interquartile) }\end{array}$ & stimulus & MP-Negative & MP-Positive & MP-Negative & MP-Positive \\
\hline IL-12 (\% positive cells) & & & & & \\
& LPS & $3.55 \pm(4.13)$ & $2.70 \pm(3.15)$ & $3.13 \pm(3.72)$ & $2.13 \pm(1.72)$ \\
& Poly:IC & $2.33 \pm(3.37)$ & $3.99 \pm(4.49)$ & $1.95 \pm(4.01)$ & $2.38 \pm(3.09)$ \\
\hline
\end{tabular}

Intracytoplasmic IL- 12 production by APC upon TLR3 and TLR4 stimulation was analysed on 43 cord blood samples; 3 I from MP-negative women and 12 MP-positive women. All comparisons between MP-negative and MP-positive women were not significant. 

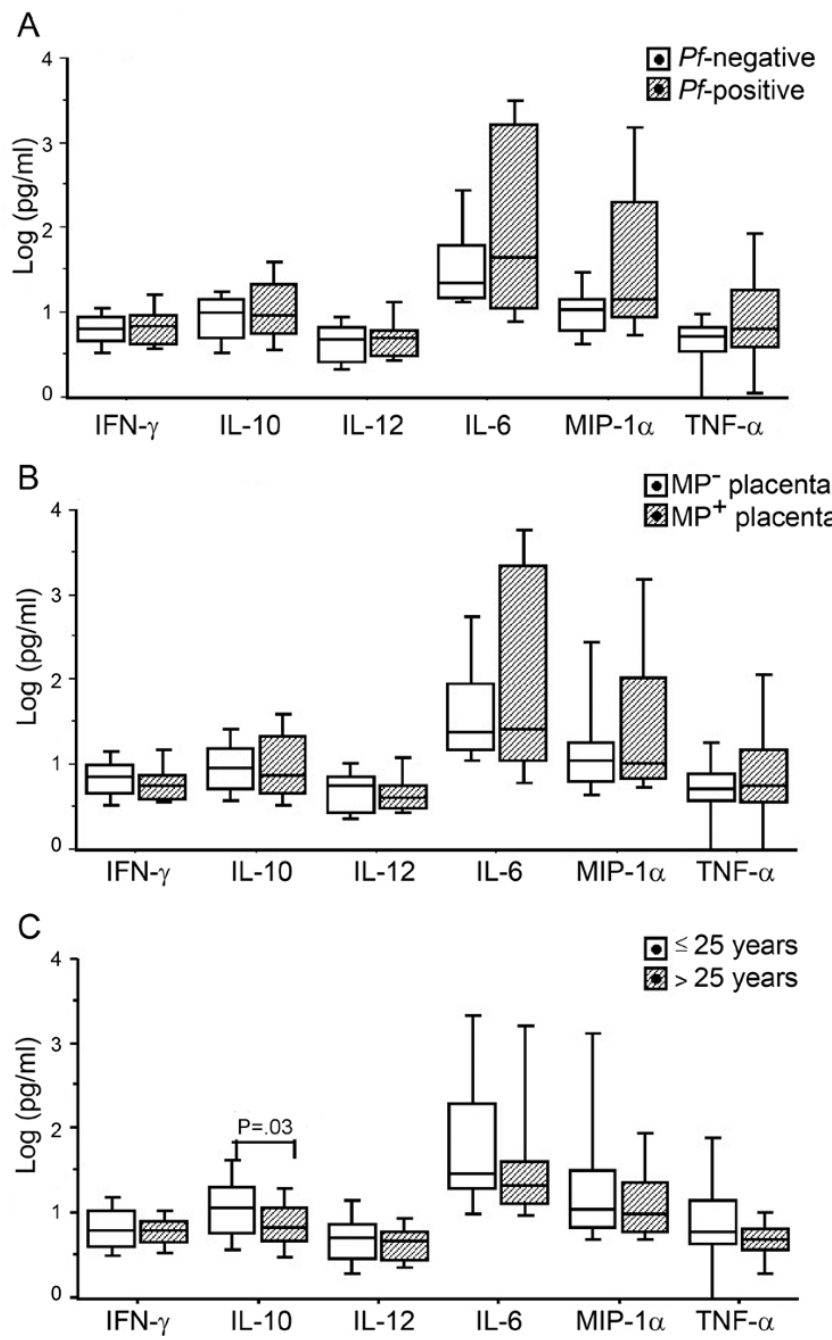

Figure 4

Cord blood cytokine levels are affected by maternal age, but not by maternal P. falciparum infection or MP accumulation in placenta. Foetal plasma samples were collected at delivery and subsequently analysed for IL-6, IL-10, IL-12, IFN- $\gamma$, TNF- $\alpha$ and MIP-I $\alpha / C C L 3$ levels. (A) Data represent median values and percentiles for 57 individuals divided in 27 P. falciparum negative mothers (Pf-negative, white bars) and 30 P. falciparum positive mothers (Pf-positive, diagonal striped bars). (B) Women were divided in 39 MPnegative mothers (white bars) and $18 \mathrm{MP}$-positive (diagonal striped bars). (C) Women were divided in 33 mothers $\leq 25$ years of age (white bars) and 24 women $>25$ years (diagonal striped bars) Values were calculated by Mann-Whitney $U$ test or $t$ test (see Subjects, Materials and Methods). The significance limit was $P<0.05$.

PDC $[25,40]$, was employed. In concordance with the findings presented in this study, MP or alternatively plasmodial DNA bound to MP activate the TLR9 pathway in human and murine PDC $[27,41,42]$. This would suggest a role for MP derived from maternal parasitic infection in
IL-6

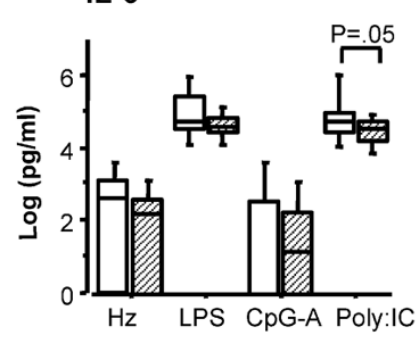

IL-12

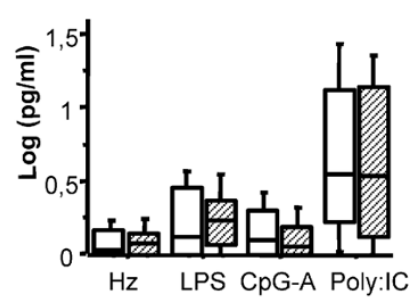

TNF- $\alpha$

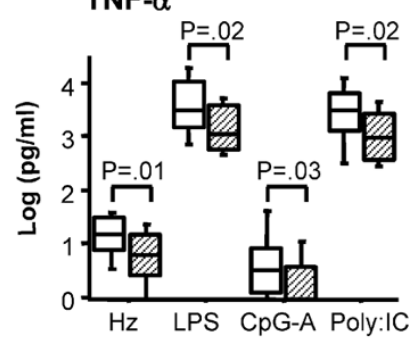

IL-10

$\square 25$ years

$\square>25$ years

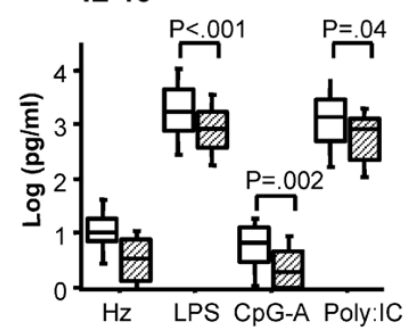

IFN- $\gamma$

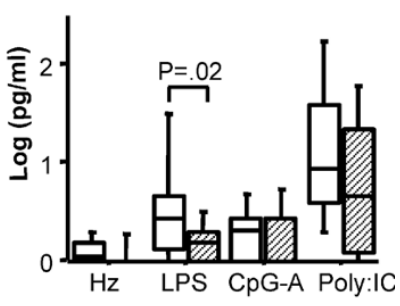

MIP-1 $\alpha$

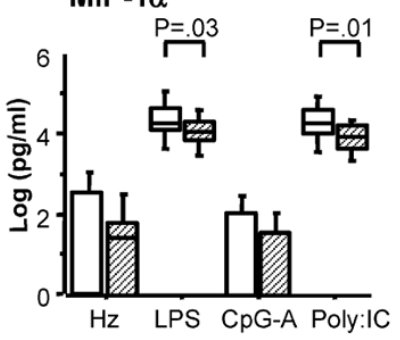

Figure 5

TLR-induced cytokine responses in foetal leukocytes are influenced by maternal age. CBMC $(2 \mathrm{million} / \mathrm{ml})$ were stimulated with synthetic $\mathrm{Hz}(5 \mu \mathrm{g} / \mathrm{ml})$, LPS $(100 \mathrm{ng} / \mathrm{ml})$, CpG-A $(3 \mu \mathrm{g} / \mathrm{ml})$ or Polyl:C $(20 \mu \mathrm{g} / \mathrm{ml})$. After 18 hours, supernatants were collected and analysed for IL-6, IL-I0, IL12, IFN- $\alpha$, TNF- $\alpha$ and MIP-I $\alpha / C C L 3$ levels. Cytokine levels in unstimulated cells were subtracted from the values shown. $\mathrm{P}$-values were calculated by Mann-Whitney $U$ test or $t$ test (see Subjects, Materials and Methods). The significance limit was $P<0.05$. Data represent median values and percentiles for 57 individuals; 33 mothers $\leq 25$ years of age (white bars) and 24 women $>25$ years (diagonal striped bars) (Data on maternal age were missing for 2 subjects, that were therefore not included in this analysis).

inducing foetal BDCA-2+ DC partial maturation and increased sensitization to TLR9 ligands. Nevertheless, only low levels of IL-10 and TNF- $\alpha$ were detected in CBMC cultures upon TLR9 stimulation. This was not unexpected given the low frequency of cells able to specifically respond to such stimulus. The biological significance of a slightly increased release of IL-10 and TNF- $\alpha$ by CBMC upon TLR9 stimulation after in utero exposure to $P$. falciparum is uncertain. 
IL-6
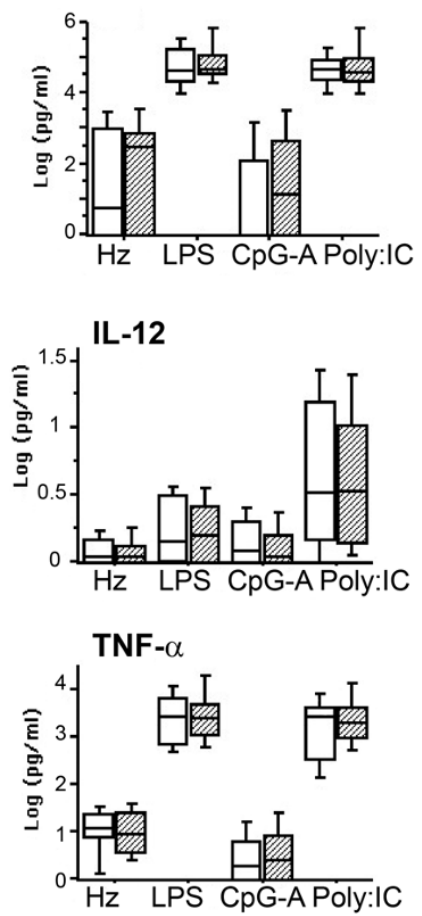

IL-10

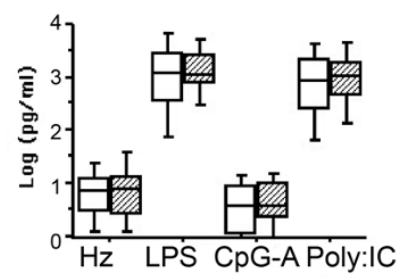

IFN- $\gamma$
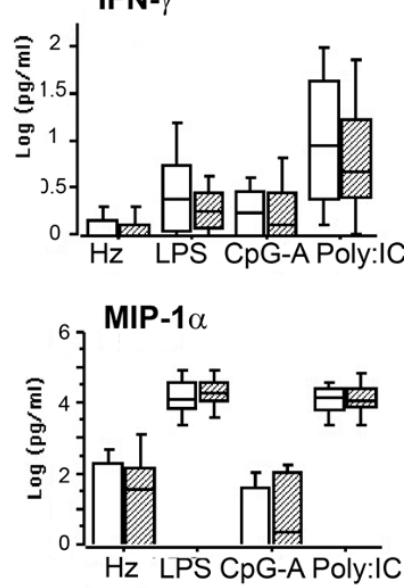

Figure 6

TLR-induced cytokine responses in foetal leukocytes are not influenced by parity. CBMC $(2 \mathrm{million} / \mathrm{ml})$ were stimulated with synthetic $\mathrm{Hz}(5 \mu \mathrm{g} / \mathrm{ml})$, LPS $(100 \mathrm{ng} / \mathrm{ml})$, CpG-A $(3 \mu \mathrm{g} / \mathrm{ml})$ or Polyl:C $(20 \mu \mathrm{g} / \mathrm{ml})$. After 18 hours, supernatants were collected and analysed for IL-6, IL- I0, ILI2, IFN- $\alpha$, TNF- $\alpha$ and MIP-I $\alpha / C C L 3$ levels Cytokine level in unstimulated cells was subtracted from the values shown. Pvalues were calculated by Mann-Whitney $U$ test or $t$ test (see Subjects, Materials and Methods). The significance limit was $P$ $<0.05$. Data represent median values and percentiles for Women were segregated on the basis of parity as 41 paucigravidae (Ist and 2nd pregnancy; white bars) and 18 multigravidae ( $\geq 3$ pregnancies; striped bars).

Notably, MP was the only indicator of maternal malaria infection that was significantly associated with partial activation of foetal DC and to amplified innate response to TLR9 ligation, while other markers of maternal parasitization at delivery such as the presence of parasites in peripheral and/or placental blood, were unrelated to DC activation in the exposed newborns. It has been recently postulated that accumulation of MP in leukocytes is a good indicator of total parasite burden, including parasite sequestration [43], and therefore we can consider accumulation of MP in placenta as a marker of high intensity of maternal malaria infection and/or of prolonged parasite exposure. In addition, accumulation of MP in placental leukocytes has been associated with increased

monocyte activation and inflammation [44]. As a hypothesis, accumulation of MP may represent a specific activation stimulus and inflammation at the placental level and this may cause partial and inadequate activation of APC in the foetal compartment.

Additionally, maternal age and parity should be taken into consideration when analysing foetal/neonatal innate immunity. Women of higher parity and increased age delivered babies in whom significantly fewer blood APC were found, but these cells exhibited an enhanced activation status. Maternal age but not parity also influenced the APC cytokine responses upon TLR stimulation, such that CBMC of offspring of younger mothers exhibited an increased ability to respond to TLR3, 4 and 9 ligands. These data are in agreement with published data on African [23] and Caucasian [45] women and suggest that maternal age and obstetric history may influence foetal/ neonatal immune parameters.

Consequences of increased maternal age and/or multiple parities in terms of neonatal responses to pathogens are poorly understood. Two recent studies indicate that the frequency of malaria episodes is higher among infants of malaria-infected multigravidae as compared to primigravidae [6,7]. The intrinsic effect of multiple pregnancies on malaria susceptibility in the offspring may be at least partially explained by our finding of a significantly reduced number of myeloid APC in foetal blood from multigravidae. How maternal age or alternatively parity can affect the number, activation status and cytokine secretion capacity of cord blood APC is presently unknown.

In conclusion, placental parasitization, as indicated by the presence of MP in placental leukocytes, is significantly associated with partial maturation of different DC subsets and to slightly increased responses to a TLR9 ligand in cord blood. As semi-maturation of DC leads to tolerance [46], such partial foetal APC activation may contribute to the altered T-cell responses often observed in newborns of mothers with PAM [5-7].

These observations advocate a possible mechanism by which PAM may modulate foetal/neonatal innate immunity. Further evaluation of APC activation and downstream T-cell responses is ongoing in a large cohort of newborns and infants from mothers with PAM to assess the impact of altered DC activation on the neonatal cellmediated immunity.

As it is known that neonatal immune responses are largely dependent on the innate branch of immunity and can be improved through selective TLR stimulation [47,48], our results should be considered in the development of effec- 
tive vaccine strategies for infants living in areas where malaria is endemic.

\section{Conflict of interests}

The authors declare that they have no competing interests.

\section{Authors' contributions}

NF: conceived the study, participated in its design and coordination, acquired the data and contributed to data analysis and interpretation and drafted the manuscript. SV: participated in the design and coordination of the study, contributed in data analysis and interpretation and drafted the manuscript. IS: acquired the data and contributed to their analysis and interpretation. VB: contributed in interpretation and statistical analysis of data and in critically revising the manuscript. SL: contributed in performing the immunoassays, and contributed in their interpretation, participated in critically revising the manuscript. RP: participated in the design of the study, in the analysis of the data and in critically revising the manuscript. AM: participated in the design and coordination of the study and in critically revising the manuscript. $\mathrm{AH}$ : participated in the design of the study, in the analysis of the data and in critically revising the manuscript. MT: participated in the design of the study, in the analysis and interpretation of data and in critically revising the manuscript. PD: participated in the design and coordination of the study and contributed to draft the manuscript. All authors read and approved the final manuscript.

\section{Additional material}

\section{Additional file 1}

Presence of $\mathrm{P}$. falciparum parasite in maternal and/or placental blood at delivery does not influence the activation status of cord blood DC and monocytes. The expression levels of HLA-DR and CD86 were measured by flow cytometry on foetal APC from $27 \mathrm{P}$. falciparum-positive (diagonal striped bars) and $28 \mathrm{P}$. falciparum-negative mothers (white bars).

Click here for file

[http://www.biomedcentral.com/content/supplementary/14752875-8-251-S1.pdf]

\section{Acknowledgements}

We thank the maternity staff of Mother and Child Lagune hospital in sample collection for their support. We are grateful to the mothers who participated in the study. We acknowledge the IRD research unit in Cotonou, especially Pepin Kounou, Sebastien Dechavanne, Bertin Vianou, Martin Amadoudji for practical support. We are also grateful to Adrian Luty for scientific support. The work received financial support from the IMEA, NATIXIS, the French Ministry of Research (FSP REFS) and from SIDA/ SAREC (Sweden). S. Louis. was the recipient of an ANRS fellowship.

Ethical issues
Informed consent was obtained from all donors. The "Faculté des Sciences de la Santé" Committee of Beninese University approved this study.

\section{References}

I. Desai M, ter Kuile FO, Nosten F, McGready R, Asamoa K, Brabin B, Newman RD: Epidemiology and burden of malaria in pregnancy. Lancet Infect Dis 2007, 7:93-104.

2. Menendez C, Ordi J, Ismail MR, Ventura PJ, Aponte JJ, Kahigwa E, Font $\mathrm{F}$, Alonso PL: The impact of placental malaria on gestational age and birth weight. J Infect Dis 2000, I 8 I: I740-I745.

3. Ordi J, Ismail MR, Ventura PJ, Kahigwa E, Hirt R, Cardesa A, Alonso $\mathrm{PL}$, Menendez C: Massive chronic intervillositis of the placenta associated with malaria infection. Am J Surg Pathol 1998, 22:1006-1011.

4. Elliott SR, Spurck TP, Dodin JM, Maier AG, Voss TS, Yosaatmadja F, Payne PD, McFadden GI, Cowman AF, Rogerson SJ, Schofield L, Brown GV: Inhibition of Dendritic Cell Maturation by Malaria Is Dose Dependent and Does Not Require Plasmodium falciparum Erythrocyte Membrane Protein I. Infect Immun 2007, 75:3621-3632.

5. Deloron P, Dubois B, Le Hesran JY, Riche D, Fievet N, Cornet M, Ringwald $P$, Cot $M$ : Isotypic analysis of maternally transmitted Plasmodium falciparum-specific antibodies in Cameroon, and relationship with risk of $\boldsymbol{P}$. falciparum infection. Clin Exp Immunol 1997, I 1 0:21 2-218.

6. Mutabingwa TK, Bolla MC, Li JL, Domingo GJ, Li X, Fried M, Duffy PE: Maternal malaria and gravidity interact to modify infant susceptibility to malaria. PLoS Med 2005, 2:e407.

7. Schwarz NG, Adegnika AA, Breitling LP, Gabor J, Agnandji ST, Newman RD, Lell B, Issifou S, Yazdanbakhsh M, Luty AJ, Kremsner PG, Grobusch MP: Placental malaria increases malaria risk in the first 30 months of life. Clin Infect Dis 2008, 47: 1017-1025.

8. Fievet N, Ringwald P, Bickii J, Dubois B, Maubert B, Le Hesran JY, Cot $M$, Deloron P: Malaria cellular immune responses in neonates from Cameroon. Parasite Immunol 1996, I 8:483-490.

9. Ismaili J, Sande M van der, Holland MJ, Sambou I, Keita S, Allsopp C, Ota MO, McAdam KP, Pinder M: Plasmodium falciparum infection of the placenta affects newborn immune responses. Clin Exp Immunol 2003, I 33:4|4-42I.

10. King CL, Malhotra I, Wamachi A, Kioko J, Mungai P, Wahab SA, Koech D, Zimmerman P, Ouma J, Kazura JW: Acquired immune responses to Plasmodium falciparum merozoite surface protein-I in the human fetus. J Immunol 2002, I 68:356-364.

II. Brustoski K, Moller U, Kramer M, Petelski A, Brenner S, Palmer DR, Bongartz M, Kremsner PG, Luty AJ, Krzych U: IFN-gamma and IL10 mediate parasite-specific immune responses of cord blood cells induced by pregnancy-associated Plasmodium falciparum malaria. J Immunol 2005, I 74: I738-I745.

12. Brustoski K, Moller U, Kramer M, Hartgers FC, Kremsner PG, Krzych $U$, Luty AJ: Reduced cord blood immune effector-cell responsiveness mediated by CD4+ cells induced in utero as a consequence of placental Plasmodium falciparum infection. $J$ Infect Dis 2006, 193:146-154.

13. Wykes MN, Good MF: What really happens to dendritic cells during malaria? Nat Rev Microbiol 2008, 6:864-870.

14. Wykes MN, Liu XQ, Beattie L, Stanisic DI, Stacey KJ, Smyth MJ, Thomas R, Good MF: Plasmodium strain determines dendritic cell function essential for survival from malaria. PLoS Pathog 2007, 3:e96.

15. Belkaid $Y$, Rouse BT: Natural regulatory $\mathbf{T}$ cells in infectious disease. Nat Immunol 2005, 6:353-360.

16. Lindstedt M, Lundberg K, Borrebaeck CA: Gene family clustering identifies functionally associated subsets of human in vivo blood and tonsillar dendritic cells. J Immunol 2005, I 75:4839-4846.

17. Piccioli D, Tavarini S, Borgogni E, Steri V, Nuti S, Sammicheli C, Bardelli M, Montagna D, Locatelli F, Wack A: Functional specialization of human circulating CDI 6 and $C D I c$ myeloid dendritic-cell subsets. Blood 2007, 109:5371-5379.

18. Levy O: Innate immunity of the newborn: basic mechanisms and clinical correlates. Nat Rev Immunol 2007, 7:379-390.

19. Adkins B, Jones $M, B u$ Y, Levy RB: Neonatal tolerance revisited again: specific CTL priming in mouse neonates exposed to small numbers of semi- or fully allogeneic spleen cells. Eur J Immunol 2004, 34:190I-1909. 
20. Eijnden S Vanden, Goriely S, De Wit D, Goldman M, Willems F: Preferential production of the IL-I2(p40)/IL-23(p/9) heterodimer by dendritic cells from human newborns. Eur J Immunol 2006, 36:21-26.

21. Encabo A, Solves P, Carbonell-Uberos F, Minana MD: The functional immaturity of dendritic cells can be relevant to increased tolerance associated with cord blood transplantation. Transfusion 2007, 47:272-279.

22. Adegnika AA, Kohler C, Agnandji ST, Chai SK, Labuda L, Breitling LP, Schonkeren D, Weerdenburg E, Issifou S, Luty AJ, Kremsner PG, Yazdanbakhsh M: Pregnancy-Associated Malaria Affects TollLike Receptor Ligand-Induced Cytokine Responses in Cord Blood. J Infect Dis 2008, 198:928-936.

23. Breitling LP, Fendel R, Mordmueller B, Adegnika AA, Kremsner PG, Luty AJ: Cord blood dendritic cell subsets in African newborns exposed to Plasmodium falciparum in utero. Infect Immun 2006, 74:5725-5729.

24. Diallo M, Aldebert D, Moreau JC, Ndiaye M, Jambou R: Decrease of lymphoid dendritic cells in blood frommalaria-infected pregnant women. Int J Parasitol 2008 in press.

25. Krug A, Rothenfusser S, Hornung V, Jahrsdorfer B, Blackwell S, Ballas ZK, Endres S, Krieg AM, Hartmann G: Identification of CpG oligonucleotide sequences with high induction of IFN-alphal beta in plasmacytoid dendritic cells. Eur J Immunol 200I, 3I:2154-2163.

26. Egan TJ: Structure-function relationships in chloroquine and related 4-aminoquinoline antimalarials. Mini Rev Med Chem 200I, I:II3-123.

27. Parroche P, Lauw FN, Goutagny N, Latz E, Monks BG, Visintin A, Halmen KA, Lamphier M, Olivier M, Bartholomeu DC, Gazzinelli RT, Golenbock DT: Malaria hemozoin is immunologically inert but radically enhances innate responses by presenting malaria DNA to Toll-like receptor 9. Proc Natl Acad Sci USA 2007, 104:1919-1924.

28. Jakobsen PH, Rasheed FN, Bulmer JN, Theisen M, Ridley RG, Greenwood BM: Inflammatory reactions in placental blood of Plasmodium falciparum- infected women and high concentrations of soluble E-selectin and a circulating $P$. falciparum protein in the cord sera. Immunology 1998, 93:264-269.

29. Briand V, Denoeud L, Massougbodji A, Cot M: Efficacy of intermittent preventive treatment versus chloroquine prophylaxis to prevent malaria during pregnancy in Benin. J Infect Dis 2008, 198:594-60I.

30. Millington OR, Gibson VB, Rush CM, Zinselmeyer BH, Phillips RS, Garside $P$, Brewer JM: Malaria impairs $T$ cell clustering and immune priming despite normal signal I from dendritic cells. PLoS Pathog 2007, 3: 1380-1387.

31. Bhattacharyya S, Cowan MJ: B7.2-/- mature dendritic cells generate T-helper 2 and regulatory $T$ donor cells in fetal mice after in utero allogeneic bone marrow transplantation. Biol Blood Marrow Transplant 2005, I I:657-67I.

32. Hochweller K, Anderton SM: Kinetics of costimulatory molecule expression by $T$ cells and dendritic cells during the induction of tolerance versus immunity in vivo. Eur J Immunol 2005, 35: $1086-1096$.

33. Schultze J, Nadler LM, Gribben JG: B7-mediated costimulation and the immune response. Blood Rev 1996, 10:1 II-127.

34. Schwartz RH: A cell culture model for T lymphocyte clonal anergy. Science 1990, 248:1349-1356.

35. Urban BC, Cordery D, Shafi MJ, Bull PC, Newbold Cl, Williams TN, Marsh K: The frequency of BDCA3-positive dendritic cells is increased in the peripheral circulation of Kenyan children with severe malaria. Infect Immun 2006, 74:6700-6706.

36. Urban BC, Mwangi T, Ross A, Kinyanjui S, Mosobo M, Kai O, Lowe B, Marsh K, Roberts DJ: Peripheral blood dendritic cells in children with acute Plasmodium falciparum malaria. Blood 2001, 98:2859-2861.

37. Urban BC, Ferguson DJ, Pain A, Willcox N, Plebanski M, Austyn JM, Roberts D): Plasmodium falciparum-infected erythrocytes modulate the maturation of dendritic cells. Nature 1999, 400:73-77.

38. Broen K, Brustoski K, Engelmann I, Luty AJ: Placental Plasmodium falciparum infection: causes and consequences of in utero sensitization to parasite antigens. Mol Biochem Parasitol 2007, | $51: 1-8$.
39. Barchet W, Wimmenauer V, Schlee M, Hartmann G: Accessing the therapeutic potential of immunostimulatory nucleic acids. Curr Opin Immunol 2008, 20:389-395.

40. Gursel M, Gursel I, Mostowski HS, Klinman DM: CXCLI6 influences the nature and specificity of CpG-induced immune activation. I Immunol 2006, I 77:1575-1580.

4I. Coban C, Ishii KJ, Kawai T, Hemmi H, Sato S, Uematsu S, Yamamoto $\mathrm{M}$, Takeuchi $\mathrm{O}$, Itagaki $\mathrm{S}$, Kumar $\mathrm{N}$, et al.: Toll-like receptor 9 mediates innate immune activation by the malaria pigment hemozoin. J Exp Med 2005, 20 I: I9-25.

42. Pichyangkul S, Yongvanitchit K, Kum-arb U, Hemmi H, Akira S, Krieg AM, Heppner DG, Stewart VA, Hasegawa H, Looareesuwan S, Shanks GD, Miller RS: Malaria blood stage parasites activate human plasmacytoid dendritic cells and murine dendritic cells through a Toll-like receptor 9-dependent pathway. J Immunol 2004, 172:4926-4933.

43. Hanscheid T, Egan TJ, Grobusch MP: Haemozoin: from melatonin pigment to drug target, diagnostic tool, and immune modulator. Lancet Infect Dis 2007, 7:675-685.

44. Diouf I, Fievet N, Doucoure S, Ngom M, Andrieu M, Mathieu JF, Gaye A, Thiaw OT, Deloron P: IL-I 2 producing monocytes and IFNgamma and TNF-alpha producing T-lymphocytes are increased in placentas infected by Plasmodium falciparum. Reprod Immunol 2007, 74: I52-162.

45. McGuckin CP, Basford C, Hanger K, Habibollah S, Forraz N: Cord blood revelations: the importance of being a first born girl, big, on time and to a young mother! Early Hum Dev 2007, 83:733-741.

46. Lutz MB, Schuler G: Immature, semi-mature and fully mature dendritic cells: which signals induce tolerance or immunity? Trends Immunol 2002, 23:445-449.

47. De Wit D, Olislagers V, Goriely S, Vermeulen F, Wagner H, Goldman $M$, Willems F: Blood plasmacytoid dendritic cell responses to CpG oligodeoxynucleotides are impaired in human newborns. Blood 2004, 103:1030-1032.

48. Wynn JL, Neu J, Moldawer LL, Levy O: Potential of immunomodulatory agents for prevention and treatment of neonatal sepsis. J Perinatol 2009, 29(2):79-88.

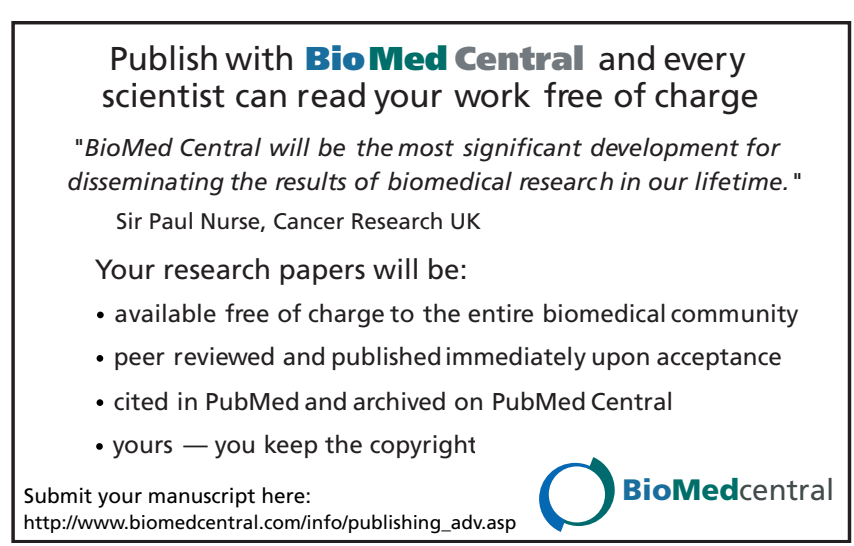

